This item was submitted to Loughborough's Research Repository by the author.

Items in Figshare are protected by copyright, with all rights reserved, unless otherwise indicated.

\title{
Distribution network reconfiguration in smart grid system using modified particle swarm optimization
}

PLEASE CITE THE PUBLISHED VERSION

http://dx.doi.org/10.1109/ICRERA.2016.7884556

PUBLISHER

(C) IEEE

VERSION

AM (Accepted Manuscript)

LICENCE

CC BY-NC-ND 4.0

\section{REPOSITORY RECORD}

Atteya, I.I., H.A. Ashour, N. Fahmi, and Dani Strickland. 2019. "Distribution Network Reconfiguration in Smart Grid System Using Modified Particle Swarm Optimization”. figshare. https://hdl.handle.net/2134/25185. 


\section{Distribution Network Reconfiguration in Smart Grid System Using Modified Particle Swarm Optimization}

\author{
Inji.I.Atteya ,Hamdy.A.Ashour \\ Electrical and Control Engineering \\ Arab Academy for Sciences and Technology \\ Alexandria, Egypt \\ Eng_inji@yahoo.com, hamdy135@gamil.com
}

\author{
Nagi.Fahmi, Danielle.Strickland \\ Electrical and Electronic Engineering \\ Aston University \\ Birmingham, England \\ n.r.fahmi@aston.ac.uk, d.strickland@aston.ac.uk
}

\begin{abstract}
One of a major characteristic of a smart protection system in Smart grid is to automatically reconfigure the network for operational condition improvements or during emergency situations to avoid outage on one hand and to ensure power system reliability the other hand. This paper proposes a modified form of particle swarm optimization to identify the optimal configuration of distribution network effectively. The difference between the Modified Particle Swarm Optimization algorithms (MPSO) and the typical one is the filtered random selective search space for initial position, which is proposed to accelerate the algorithm for reaching the optimum solution. The main objective function is to minimize the power losses as it represents waste of operational cost. The suggested method is tested on a 33 IEEE network using IPSA software. Results are compared to studies using other forms of swarm optimization algorithms such as the typical PSO and Binary PSO. 29.68\% of losses reduction has been achieved during a less computational time.
\end{abstract}

Keywords-Smart Grid; Distribution System; Distributed Network Reconfiguration; co $_{2}$ emmissions; Modified Particle Swarm Optimization .

\section{INTRODUCTION}

The uncertainty in fuel cost, the ageing of most existing grid, the lack of utilities' supply capacity to respond to the increasing load demand, and the lack of automatically power restoration, is accelerating the need to modernize the distribution network by introducing new technologies, putting the smart grid (SG) on spot. SG is a network that uses information, cyber secure, communication technologies, and computational intelligence to create an automated and distributed advanced energy delivery network to achieve a safe, reliable, efficient, and sustainable system. It coordinates the need and capabilities of all generators, grid operators, end users, and electricity market stakeholders to operate all part of the system as efficiently as possible, minimizing costs and environmental impacts while maximizing system reliability, and stability [1].

The integration of Renewable Energy Resources (RES) and Energy storage (EES) in distribution system, which justify the bidirectional flow of power in SG, is not only the major requirements of $\mathrm{SG}$, but also smart metering, monitoring and communication systems are required to gather the power system consumption information, send it via wireless communication network to electric utilities to manage and analyze the data for optimum decision making for both utilities and costumers. These requirements add more complexity on power system and dictate the necessity of having a smart protection system that should be predictive enough to expect the failures, automatically isolate the faults after detection, and select the optimum configuration to avoid outage and supply the consumers based on intelligent algorithms to ensure power system reliability [2].

A Distribution system consists of many interconnected mesh circuits, operated as radial, linked by breakers. There are two types of switches: sectionalizing switches which are normally closed and tie line switches which are normally open. Distributed network reconfiguration (DNR) is the process of changing the structure of the distribution network by changing the status of the sectionalizing and tie switches to maintain the radial topology [3]. There are three stages for DNR [4]:

1. Network Data Collection: This stage includes lines impedances, load demand, and available generation.

2. Network Organization: this stage includes the study of the different probabilities due to the change of switches states based on mathematical optimization algorithms.

3. Load Transfer: This is the implementation of the second stage via remote control switches.

In this paper, DNR definition and stages are explained in section I. The reasons for considering DNR a complex optimization problem are stated, and the mathematical formulation for DNR objectives and constraints are surveyed in section II. The 33 IEEE network is selected to be a case study. Network data and the DNR problem formulation are stated in section III. The proposed Modified Particle Swarm (MPSO) included in section IV, is explained and the difference between the suggested method and the original PSO are highlighted. The Results are included in section $\mathrm{V}$ proving the effectiveness of the technique.

\section{DNR MATHEMATICAL DESCRIPTION}

\section{A. DNR Objectives and Constraints}

DNR is considered highly complex, nonlinear, discrete, combinatorial, stochastic optimization problem [4, 5]. The non-linearity of this problem is due to the electrical equipment and the power electronic devices used in the network [4]. Its complex combinatorial nature is due to the large probability obtained by changing the switches in order to find the optimum configuration to realize the objective function within the constraints [6]. Its stochastic nature is due to the 
continuous change of demand. Its discrete nature is due to the change of the state of switches between on and off. Therefore, this problem could be described mathematically as a hardNon-Polynomial (NP) optimization problem [4, 6].

The main features of DNR are highlighted during emergency (a fault), as a part of the system should be isolated to minimize the number of affected consumers by feeding them from another feeder. DNR merits do not serve the system only in contingency condition but also, could improve the system reliability and efficiency by reducing the line losses and protecting them from over loading through load balancing. Also, it could facilitate the integration of renewable energy especially in peaks, thus reducing the generation cost. Previous researches can be classified mathematically and regrouped in figure 1 surveying all the objective functions and the constraints for DNR problem. It should be noted that the losses reduction gained a great deal of attention as it brings unnecessary operational cost. Moreover, the power losses occurred in distribution system, are too high compared to that of generation and transmission due the low voltage level and the high current passing through the distribution lines. For this reason, losses reduction is selected to be the main objective for DNR in this research.

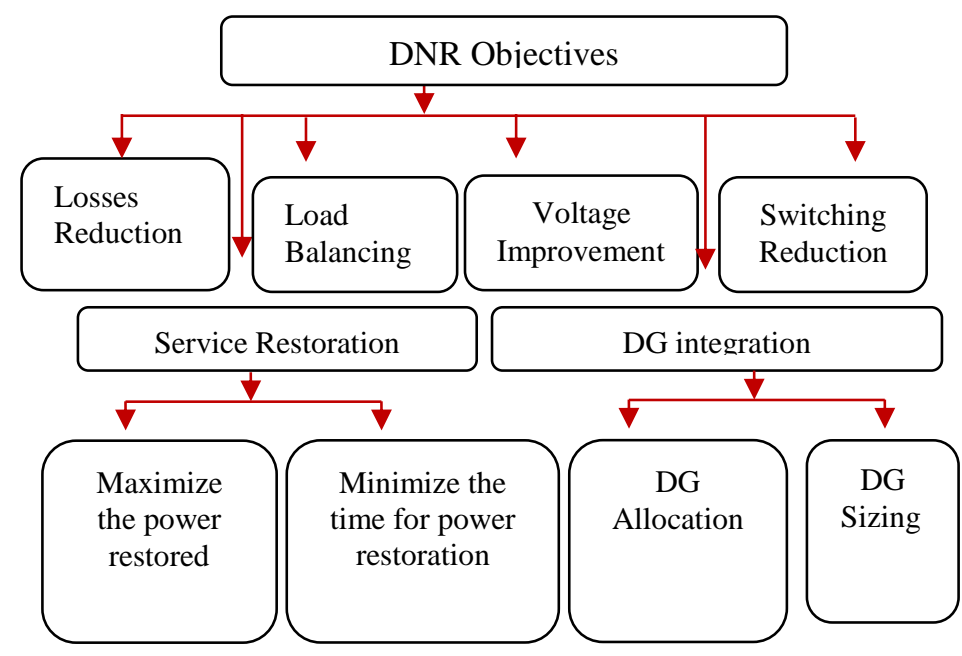

(a)

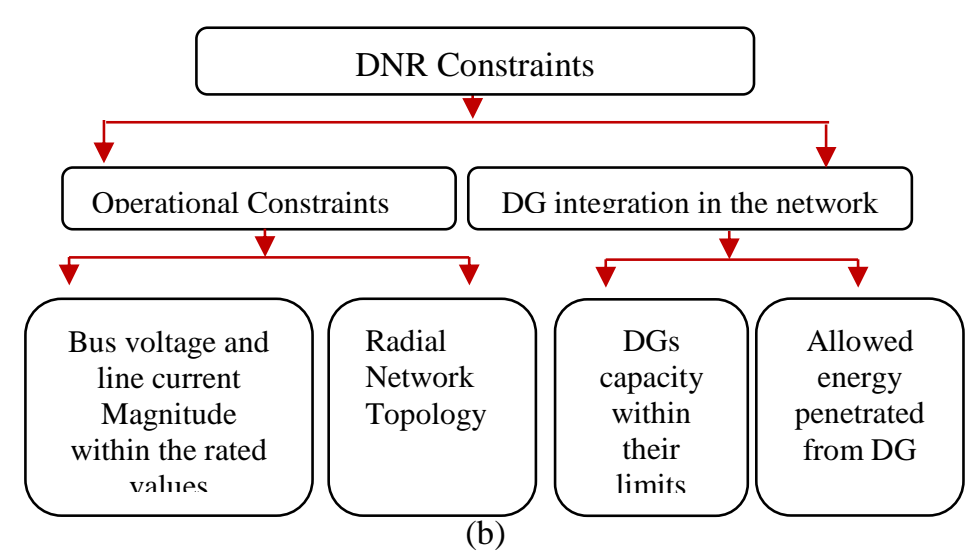

Figure 1 DNR objectives and constraints

\section{B. DNR Optimization algorithm Classification}

In this paper, DNR Optimization techniques are classified into three categories: Heuristic, Meta heuristic and Mathematical algorithms. Due to the inaccuracy of heuristic algorithms and the complexity of the mathematical ones, most of recent researches focuses on Meta heuristic techniques or hybridize them for better performance.

Heuristic techniques are knowledge based techniques; in other words, they select the optimum configuration based on operational experiences [7]. Although they are fast to solve the DNR problem, they achieve an approximate solution rather than a global optimum one. Meta heuristic techniques are probabilistic algorithms used usually in power system operation and planning to deal with uncertainties by modeling the stochastic factors in power systems such as random outages of components and uncertain variation in loads and weather conditions [8]. They achieve global optimum solution, but the computational time is too high due to their probabilistic nature and their random selection which makes their convergence speed slower [9].These algorithms are based on artificial intelligence (AI). They follow a set of nature inspired methodologies that simulate biological phenomena, and represent them into computational tools to address complex problems hard to be solved by traditional approaches. They are based on population search which means that many entities are simultaneously sent in parallel to solve the same problem [10]. The success of this method is mainly due to the possibility of obtaining results much better than heuristic algorithms. The most common AI techniques addressing the losses reduction used for DNR problems are surveyed in this section and regrouped in figure 2.

Simulating Annealing (SA) was proposed by Kirkpatrick, Gelatt and Vecchi in 1983[11], based on the cooling process of a melting metal slowly cooled to solidify in its minimum energy state. Song Nie et al. used this algorithm to study the impact of distributed generations on network reconfiguration during normal and post fault operation [12]. An IEEE 33 bus network was used to evaluate the proposed technique Although SA algorithm could achieve optimal solutions, it can be very time consuming and its performance depend on initial parameter [13]. Furthermore, the complexity of the cooling parameters could be considered a significant disadvantage, as there is no general base to select the best parameters for a given problem [14]. For these reasons, this algorithm is not suggested for real implementation until to be modified. ANN is another based artificial intelligent technique used for DNR applications. Few researches such as [15, 16] used this technique due to the large offline training time which could be a major problem facing the large-sized real networks. Therefore, this algorithm is not suggested for online operation, although it can achieve optimum configurations. Another new, simple, meta-heuristic technique, Music Based Harmony Search (MBHS), is presented by Geem et al. in 2001 [17].It was inspired by the reproduction of musicians' behaviour during playing their musical instruments which represent the population to obtain certain pleasing harmony (the global solution required). 


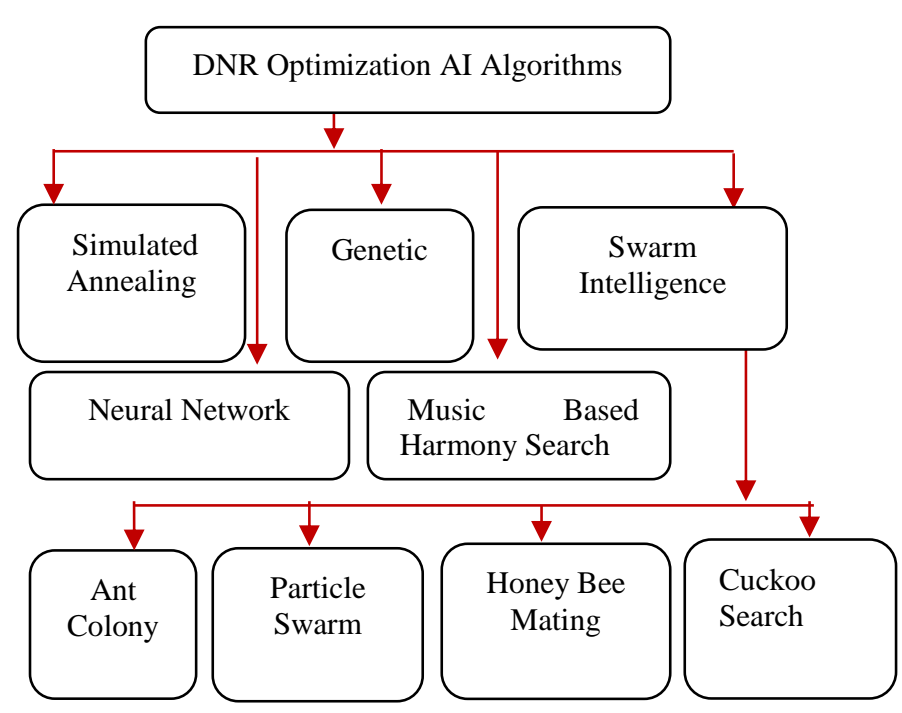

Figure 2 Most Common DNR Algorithms

In [18], the author applied this technique onto two distributions network: 33 and 69 IEEE buses through different loading conditions for losses reduction, proving the effect of DG installation on voltage and losses reduction improvement. In [19], the authors used an improved version of the technique using variable values of harmony search parameters. The suggested technique has been validated on a 33 busses IEEE network and compared to other Meta heuristic techniques such as traditional HAS, GA, RGA and ITS. It was found that, IMBHS takes less number of iterations to reach the optimal solution compared to all mentioned techniques, so that this technique is recommended in emergency service restoration problem. A self-adaptive harmony search Algorithm (SAHSA) was proposed by H.Savari et al. in 2010, by adding another step in the traditional HSA to improve the accuracy and the convergence rate on one hand, and to reduce the defect of initial parameters across the problem [20]. Unlike the previous researches $[18,19]$ where HSA was used to reduce the active power losses through network reconfiguration, D. Rani has suggested the SAHSA for both active and reactive power losses [21]. The proposed method is tested on 33 and 69 IEEE busses, and compared to MHSA. It was found that both techniques got the same value of active and reactive power losses, but the SAHSA reached these optimal results in less number of iterations. Also, the worst and the average fitness values found by SAHSA are better than MHSA. It was noticed that HAS passed through different stages from the traditional version to the self-adaptive form to modify the accuracy and reducing the number of iterations to achieve the optimum fitness value. Therefore, it is suggested in real time applications such as load balancing and network restoration. However, the addition of another step to improve the search space has an impact on the time taken compared to other algorithms.

Swarm Intelligence optimization techniques are another Meta heuristic algorithm family, based on social behaviour of swarming animals such as birds, ants and fishes. This category includes Ant Colony Optimization technique (ACO) proposed by proposed by M. Dorigio in 1992 in his $\mathrm{PhD}$ thesis [22], Particle Swarm Optimization (PSO) suggested by J.Kennedy and R.Eberhart in 1995 [23], Honey Bee Mating Optimization (HBMO), and finally Cuckoo Search Optimization Algorithm (CSA) developed by developed by Yang and Deb in 2009[24]. Many researches were carried out using PSO technique for optimum network reconfiguration considering the presence of DGs addressing the real losses reduction [25, 26, and 27]. AW.Dahalan et al. studied the power losses reduction and voltage improvement using PSO[26], validating his technique on a 33 IEEE network to prove its effectiveness, while in [27], the author tested the approach on a CIGRE distribution network including different DGs forms( such as wind turbines, photovoltaic, storage batteries and fuel cells) in order to improve the service reliability. Although PSO is a powerful simple algorithm, it was initially designed only for continuous functions not for discrete ones. This in turn pushes J.Kennedy and R.Eberhart to modify their algorithm and introduce a binary version of particle swarm technique (BPSO) in 1997[28].Significant researches are now shifting from single to multi objective function. For this reason, multi objective particle swarm was developed in 2004 by CoelloCoello [29]. A research has been carried out by A.Arya et al., for optimum distribution configuration, where a multi- objective particle swarm (MOPSO) has been used for maximum power restoration, load balancing and minimizing the switching operation and bus voltage deviation [30]. The author validated his algorithm on a 10 IEEE bus to prove the effectiveness of the approach. Both authors in [26, 30] agreed that PSO is better than GA in computational time and the number of iterations to achieve the optimum solution. Another research was presented by S.Tuldhar et al. for multi objectives functions dynamic reconfiguration problems proposing a Non Dominant Storing particle Swarm algorithm (NSPSO) [31] .The key difference in this study, that the author considered the uncertainty of both the generation of renewable energy resources and the variation of load demand. And here 33 IEEE systems is used, to test 3 cases studies and to compare between static and dynamic reconfiguration. In order to improve the computational time and the convergence characteristic, a simple modification to BPSO has been proposed in [32] by improving the search space and changing the sigmoid transformation rules used in binary form to limit the velocity between 0 and1, introducing a selective particle Swarm Algorithm (SPSO). A.Tandon and D.Saxena studied the optimum configuration considering the losses reduction to compare between the BPSO and SPSO [33] by testing their approach on a 33 and 69 IEEE bus network. Distribution losses, voltage profiles, number of switching, computational time and the convergence rate were the main comparative points. Results concluded that the SPSO outperformed over BPSO in losses reduction and voltage improvement. Overall, PSO is simple robust technique suggested by many references for DNR optimizations including distributed generations as it could support multi objective functions. Furthermore, the computational time and the convergence rate could be 
considered significant features of this approach. In this paper, a modified particle swarm optimization (MPSO) is suggested based on a filtered random selection technique for initial positions. This modification has been proposed to reduce the computational time required to get the global optimum solution.

\section{CASE STUDY}

In this section, the 33 IEEE networks, $12.6 \mathrm{kV}$, shown in figure 3 , is selected for optimum configuration for losses reduction. This network is specially used for comparing the results as it was studied in many previous researches. Interactive Power System Analysis (IPSA) tool is used for distribution network simulation and load flow calculations using python programming language.

\section{A. Network Description}

The 33 IEEE network consists of 37 branches, 32 normally closed switches (sectionalizing switches) and 5 normally open switches (Tie line switches). The system load is assumed to be constant. The initial tie lines switches of the network are from bus 33 to bus 37 before any reconfiguration. The total number of loops that should be formed by closing the tie switches is 5 loops, and then the dimension of the search space. The system load is $3,715 \mathrm{~kW}$ and $2300 \mathrm{kVAr}$. The network line data are illustrated in the appendix [34].

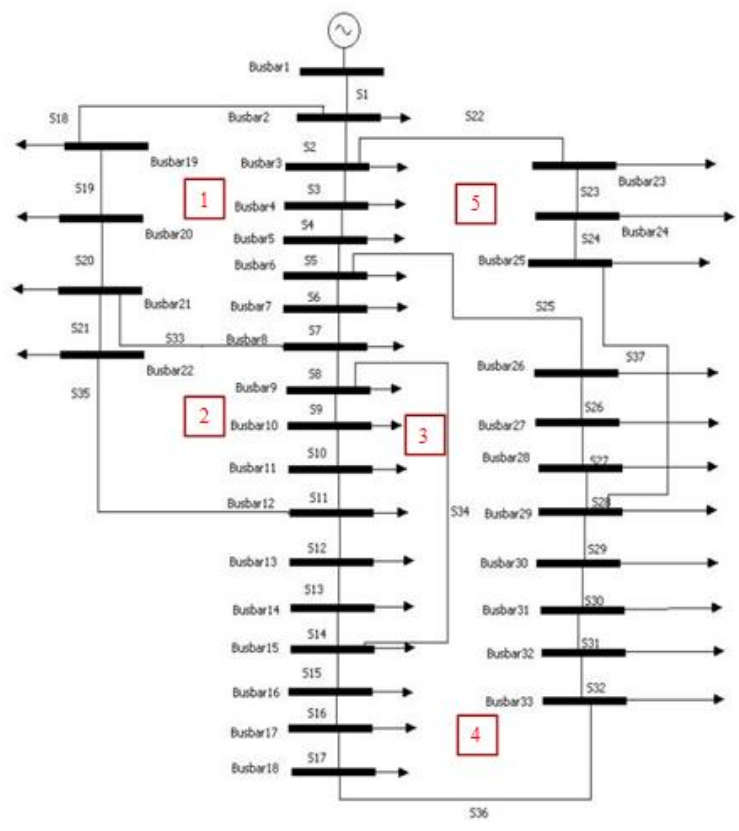

Figure 333 IEEE Network (IPSA window)

\section{B. Problem Formulation}

In this research, line losses minimization during operation is the objective function used for DNR optimization problem and could be described as:

Where :

$$
\text { Min Power } \text { losses }=\sum_{j=1}^{n}\left(I_{j}^{2}\right) R_{j}
$$

$\mathrm{j} \quad$ is the branch number

$\mathrm{N} \quad$ is the total number of branches

$\mathrm{I}_{\mathrm{j}} \quad$ is the current at branch $\mathrm{j}$

$R_{j} \quad$ is the resistance at branch $j$

\section{Constraints}

Three constraints are considered for optimum losses reduction:

1. Node voltage limit

The bus voltage magnitude should be within the permissible limits to maintain power quality. The minimum and the maximum values of the voltage are chosen to be 0.9 and 1.0 respectively.

$$
V_{\text {min }} \leq V_{\text {bus }} \leq V_{\text {max }}
$$

2. Feeder capacity limit

The magnitude of the feeder's branch current $\left(I_{j}\right)$ should not exceed the maximum value of the allowed current passing in the branch $\left(\mathrm{I}_{\max }\right)$ eliminating the insulation failures assuming that thermal limits are achieved.

$$
\mathrm{I}_{\mathrm{j}} \leq \mathrm{I}_{\max }
$$

3. Maintain the radial topology

In order to maintain a simple, inexpensive operation and protection of distribution power grid, radial configuration is preferred. It is stated that each loop should contain a tie line and a corresponding sectionalizing switches. Thus, to retain a radial network structure, when a tie is closed in a loop, only one switch should be open in the same loop [30]. To retain this topology, the following criteria should be considered:

1. The total number of main loops obtained by closing all the ties:

Where:

$$
N_{\text {main loops }}=\left(N_{b r}-N_{\text {bus }}\right)+1
$$

$N_{b r}$ is the total number of branches

$N_{\text {bus }}$ is the total number of buses

2. The total number of sectionalizing switches

$$
N_{b r}=N_{b u s}-1
$$

3. The total number of tie switches should be the same as the number of main loops.

4. The elements' selection of each loop

The 33 IEEE network is divided into 5 loops, including 5 tie switches and 32 sectionalizing switches; the members for each loop are illustrated in figure 3 , based on [33]. It should be noted that the switch S1 is not included in any loop which means that it could not be disconnected, as it connects between the main supply and the network loops. The switches common between more than one loops, are stated only one time, and this to eliminate the repeated switches inside a configuration. For example, S2 is common between loop (1) and loop (5), but it is an element only in loop (1). In this research, the total search space for initial radial configurations is 16128 configurations calculated based on the tree diagram 
probability method which generates all the possible configurations that include only one tie switch from each of the main loops. Fig.4 shows an example of how tree diagram could be used to generate a configuration such as (S8, S2, S12, $\mathrm{S} 15$, and S22). The first element of each of the 4 loops should pass through all the elements existing in the $5^{\text {th }}$ loop. Another configuration could be (S8, S2, S12, S15, and S23) and so on until (S8, S2, S12, S15, and S37).

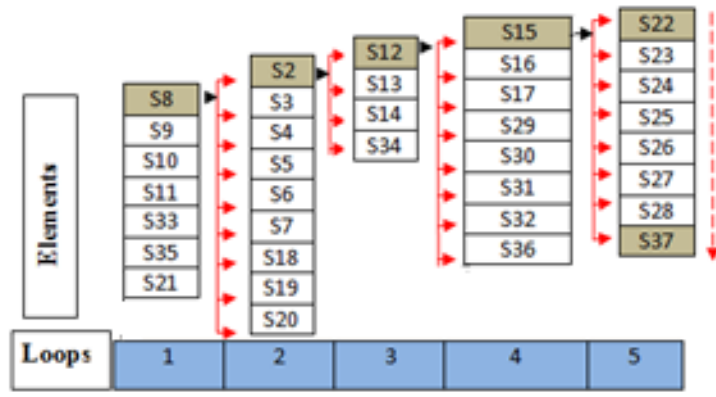

Figure 4 Example of a radial configuration in the search space by tree diagram

\section{DNR USING MPSO ALGORITHM}

\section{A. Typical PSO Method}

The typical PSO is inspired by the ability of a group of some species of animals to work as a whole in a given area searching for corn. This seeking behavior is validated through equations in a real valued search space. Particles move through the search space adjusting their positions and their velocities according to their own experience and to their neighboring particles experience in order to find the optimal solution based on equations (6) and (7). The searching space is composed of all the possibilities that could represent a solution for the fitness function. This in turn explains the high processing time used to perform the calculations. The analogy between the biological system and the engineering case study is explained in the next section.

$$
\begin{aligned}
V_{i}{ }^{K+1}= & w * V_{i}^{k}+c_{1} * \operatorname{rand}_{1} *\left(P_{\text {best }_{k}}-X_{i_{k}}\right)+ \\
& c_{2} * \operatorname{rand}_{2} *\left(G_{\text {best }_{k}}-X_{i_{k}}\right) \\
X_{i}{ }^{k+1}= & X_{i_{k}}+V_{i}^{k+1}
\end{aligned}
$$

Where:

$w$ the inertia weight, it is a decreasing function calculated according to equation (9).

$V_{i}{ }^{k} \quad$ is the velocity for the particle $(i)$ for the iteration $(k)$

$c_{1}, c_{2} \quad$ Acceleration variable usually set to 2.0

rand $_{1}$, rand $_{2}$ a random number from 0 to 1

$P_{\text {best }}$ Best position for particle (i) based on its own experience.

$G_{\text {best }}$ Best position achieved by the entire particles in the swarm

$$
w^{k}=\frac{\left(w_{\max }-w_{\min }\right)}{\text { iter }_{\max }} * \text { iter }
$$

\section{Where}

$w_{\max }$ is $0.9, w_{\min }$ is 0.4 , iter $_{\max }$ is the total number of iterations and iter is the current iteration.
1. Particle's position and velocity Representation for PSO in a DNR problem

The individual particle $(i)$ in this case is composed of a set of the tie switches $\left(S_{1, \ldots .} S_{n}\right)$ that are to be opened in a radial system, where $(n)$ is the size of the particle, in a swarm of (S) particles. It should be noted that the particle's size is the same size of tie switches in a system. The position of the particle $\left(X_{i}\right)$ is the index of the tie switch per loop. For example; $X_{i}=$ [S33, S34, S35, S36, S37], means that (S33) is the first switch selected to be opened for loop (1), while the second switch to be opened is (S34) and etc. It should be stated that the particles positions should be positive numbers and integer as they represent switches indices. In this research, the noninteger numbers are rounded up or down to the nearest digit, for example S3.2 is S3. The initial velocities are assumed to be zeros.

\section{2. $P_{\text {best }}$ and $G_{\text {best }}$ representation in a DNR problem}

In PSO, during each iteration, $P_{b e s t}$ and $G_{b e s t}$ are updated and recorded based on the objective function. In other words, $P_{b e s t}$ is the configuration realizing best fitness function (losses reduction) for the same particle; while $G_{\text {best }}$ is the configuration achieving best losses reduction for all the particles for one iteration.

\section{B. Implementation of MPSO in Network Reconfiguration}

In this research, MPSO is used for optimum losses reduction. The main difference between the original PSO and the MPSO is the filtered random selective search space in the initial position, which improves the searching capability of the particles in less computational time by neglecting the infeasible particles based on the current and the voltage constraint after load flow calculations, and this in turn accelerate the algorithm. MPSO flow chart is represented in fig.5 and fig. 6 .

\section{Filtered Random Initial Position Selection:}

Although PSO is based on a random initial selection for positions and velocities, in this paper, a filtered random selection procedures illustrated in fig.7, is suggested to control the initial positions of the particles. It should be noted that implementing such modification accelerated the particles search and in turns reduced the excitation time of the overall program for reaching the global optimum solution.

\section{Position Control}

After updating the particles using equation (7), some positions could exceed the total number of switches in the existing network, (S37 in this network), or could be negative number, which is illogical. In previous version of swarm, these infeasible positions are discarded, thus losing some probabilities. Thus, to maintain a feasible search space, a position control algorithm has been suggested in [35], and is 


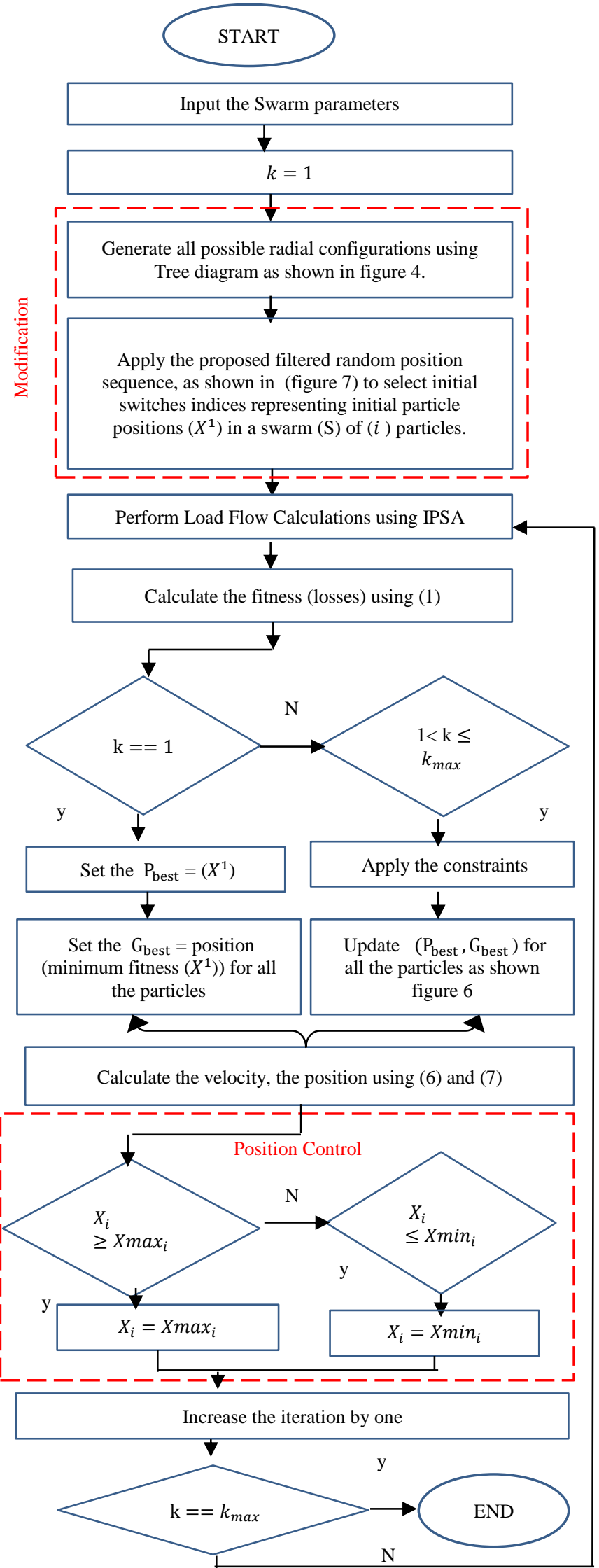

Figure 5 MPSO Flow Chart

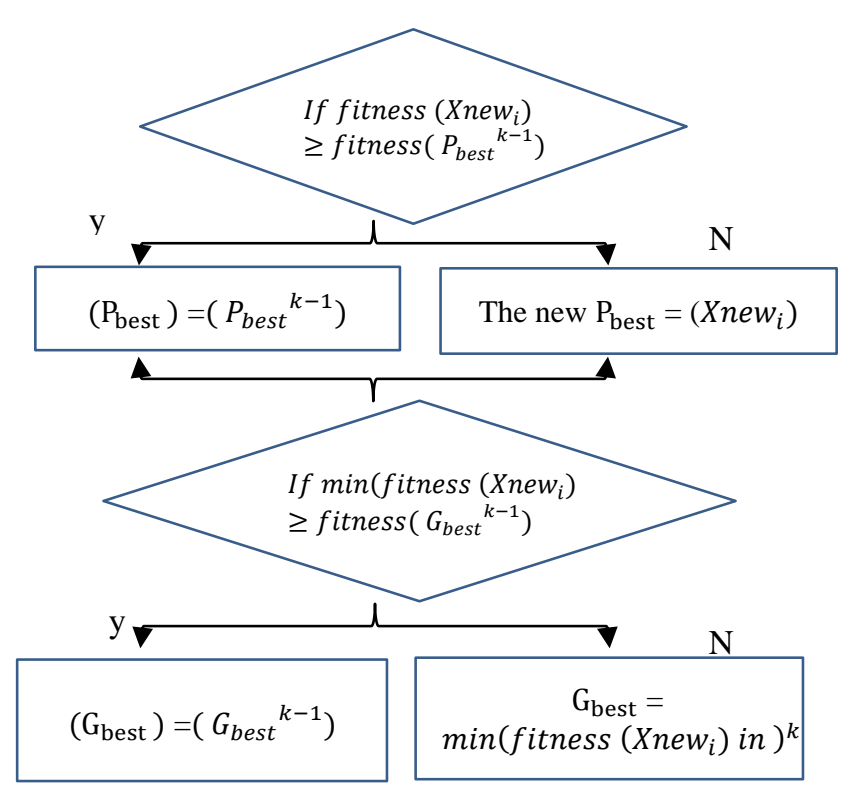

Figure 6 Pbest and Gbest Update

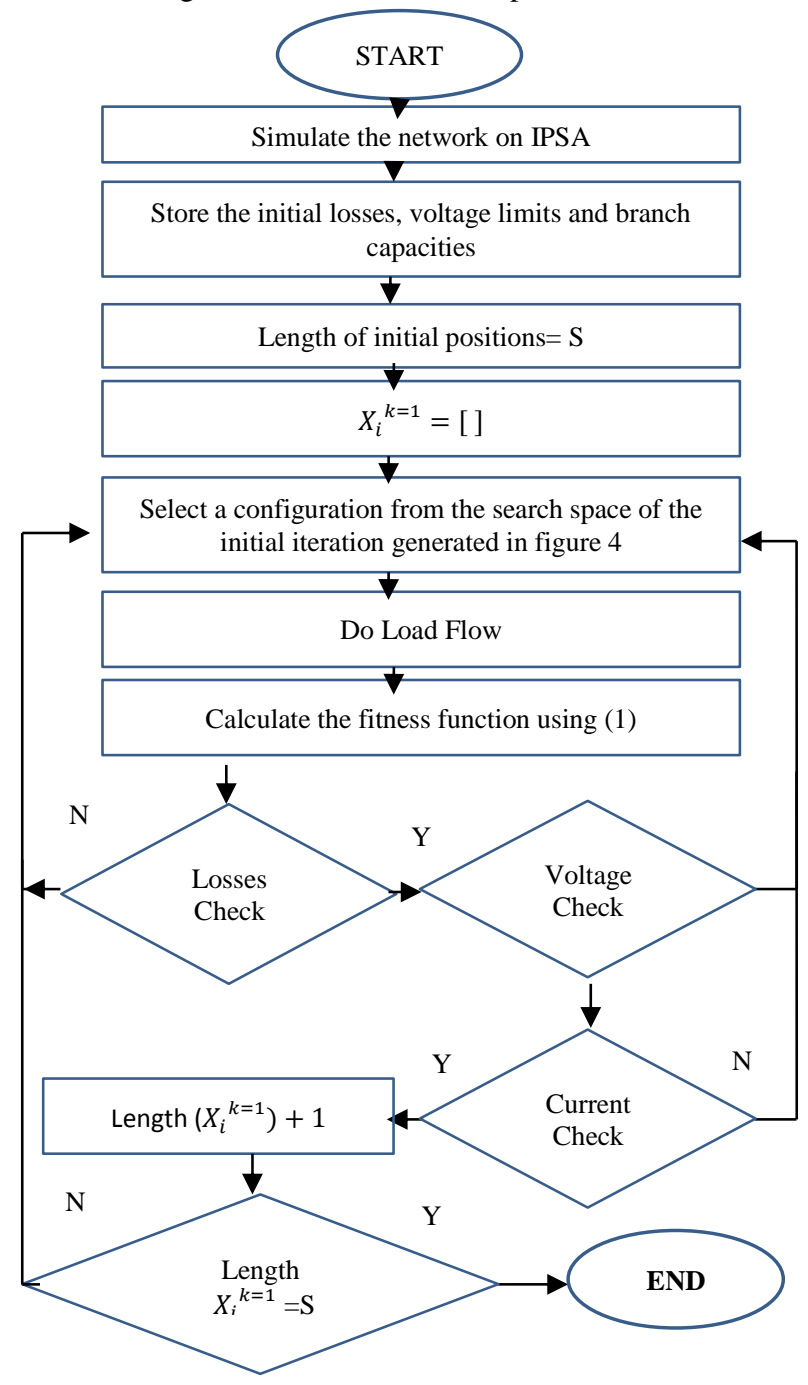

Figure 7 Filtered randominitial position sequence 
applied in this paper. Position Control's procedures are illustrated in fig.5. Although, this algorithm retains all the particles in the search space, it could duplicate some switches in the same particle position, and violate the tie switch number conditions, which are calculated to be 5, and only in this case the particle should be discarded.

\section{Conversion Condition}

One of the main keys to obtain an accurate algorithm is to initialize the software after each losses calculation for each position. It was found that, when the algorithm changes the positions without a reference configuration, it gives inaccurate results leading to a long computational time. For this reason, a known configuration should be selected to be an initial attempt before each trial. In this study, the initial attempt is meshing the network after each trial. For example, the initial tie configuration for the 33 IEEE systems is [S33, S34, S35, S36, and S37]. It could be considered as a particle position. Thus initially, the network is totally closed and then after opening the tie configuration, the network is closed again until selecting other tie configurations in the swarm size.

\section{IPSA Software for 33 IEEE network Validation}

Interactive Power System Analysis (IPSA) is a software tool developed specifically for power system design and operation applications providing a fast and accurate analysis of electrical power systems through an intuitive user interface [36]. Analysis options include load flow, fault level and the software can be driven by Python programing language. Fig. 8 shows the mechanism for IPSA software, where the designed python script including the proposed optimization algorithm (shown in fig.5, fig.6) is called by IPSA to control the inputs of the network (as illustrated in Appendix) simulated in fig.3.

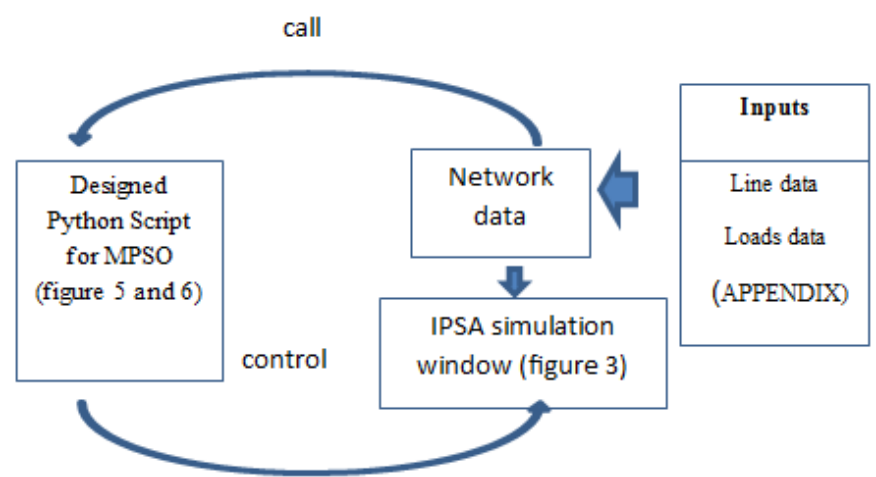

Figure 8 IPSA software diagram

Table 1 MPSO Parameters

\begin{tabular}{|c|c|c|c|c|}
\hline PSO & $\begin{array}{c}\text { Swarm } \\
\text { Size }\end{array}$ & $\begin{array}{c}\text { Weighting } \\
\text { factor }\end{array}$ & $\begin{array}{c}\text { Acceleration } \\
\text { constant } \\
\text { Carameters } 1, \mathrm{C} 2\end{array}$ & $\begin{array}{c}\text { Initial } \\
\text { velocity }\end{array}$ \\
\hline & 50 & {$[0.9-0.4]$} & 2.0 & 0.0 \\
\hline
\end{tabular}

\section{RESULTS AND DISCUSSION}

\section{Losses Reduction}

The 33 IEEE network is simulated using IPSA software for losses, load flow and optimization technique implementation. Developed software has been designed to implement the (MPSO) using PYTHON 2.7.8, on a $2.4 \mathrm{GHz}$, core (TM) i75500CPU with 8.0- GB RAM. In this paper, the initial losses are $193.6 \mathrm{~kW}$. The maximum and minimum bounds for voltage magnitude are set to 0.9 to 1 .pu. Load flow results are compared to previous research for validation [37]. The initial ties switches were from line 33 to 37 . After applying the suggested algorithm, the losses are reduced to $136.36 \mathrm{~kW}$ reducing $29.68 \%$ of the initial value. MPSO parameters used during simulation for reconfiguration are summarized in table 1. Due to the stochastic nature of swarm algorithms, 50 runs are performed in order to find the best number of particles and iterations for optimum fitness function. In each trial, the best, the worst and the average value of the fitness function (the losses) are recorded as well as the computational time, as illustrated in table 2 . In additions, the mode, which represents how frequent the best losses occurred during one trial, is recorded. It was found that a population size of 50 particles during 25 iterations is sufficient for finding the optimal losses value. Fig. 9 shows the convergence characteristic for the proposed algorithm.

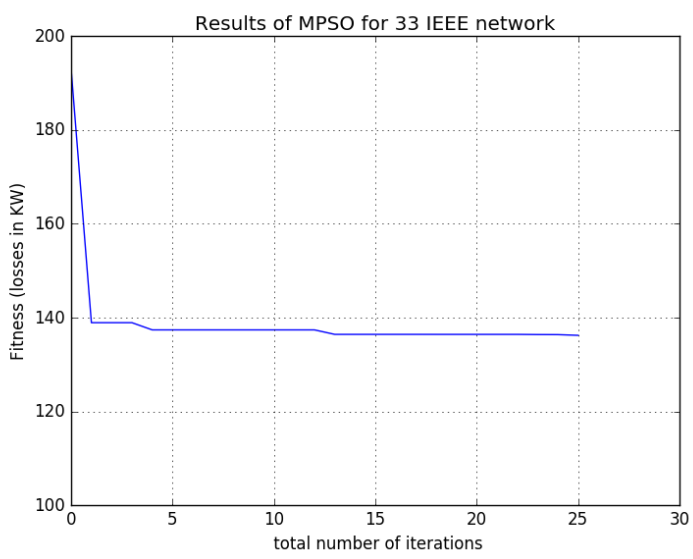

Figure 9 Fitness Function convergence using MPSO

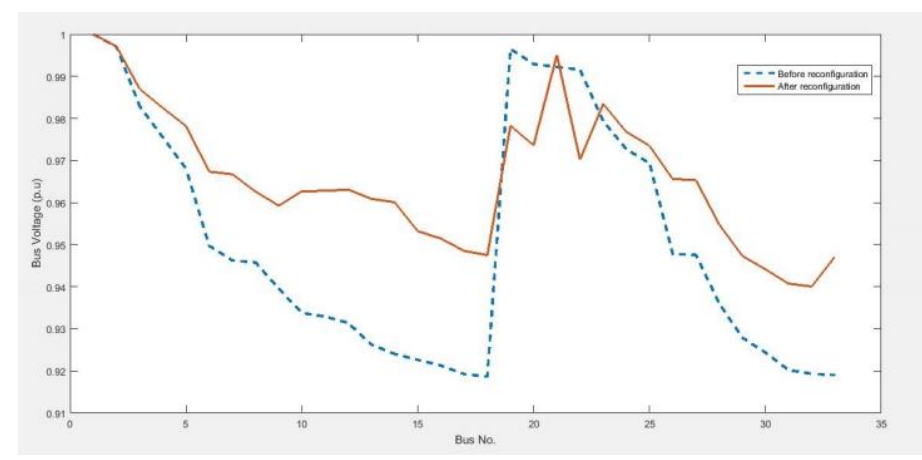

Figure 10 Voltage Profile Improvements 


\begin{tabular}{|c|c|c|c|c|c|c|}
\hline Particles & iterations & $\begin{array}{c}\text { Best } \\
\text { losses }\end{array}$ & $\begin{array}{c}\% \\
\text { Mode }\end{array}$ & $\begin{array}{c}\text { Worst } \\
\text { losses }\end{array}$ & Average & time \\
\hline 50 & 25 & 136 & $52 \%$ & 143 & 135 & 17.5 \\
\hline
\end{tabular}

\section{Voltage Improvement}

A significant improvement in voltage profile is observed after applying the suggested algorithm. The minimum bus voltage after reconfiguration rise to 0.94 at bus 32 , from 0.918 at bus 18 initially before reconfiguration, as shown in fig. 10 .

\section{Table 2 33IEEE network using dif ferent Swarm Algorithms}

\begin{tabular}{|c|c|c|c|c|}
\hline & Algorithm & Switches & $\begin{array}{c}\text { Losses } \\
(\mathrm{kW})\end{array}$ & $\begin{array}{c}\text { Min. } \\
\text { Volt }\end{array}$ \\
\hline $\begin{array}{c}\text { without } \\
\text { Reconfiguration }\end{array}$ & Initial & $\begin{array}{c}33,34,35, \\
36,37\end{array}$ & 193.3 & 0.918 \\
\hline \multirow{4}{*}{$\begin{array}{c}\text { After } \\
\text { Reconfiguration }\end{array}$} & PSO & $\begin{array}{c}33,28,34,8, \\
17\end{array}$ & 149.8 & 0.931 \\
\cline { 2 - 5 } & $\begin{array}{c}125] \\
{[26]}\end{array}$ & $\begin{array}{c}7,10,28 \\
14,32\end{array}$ & 140.5 & 0.941 \\
\cline { 2 - 5 } & BPSO & $7,9,14,28,3$ & 139.8 & 0.941 \\
& {$[33]$} & 2 & & \\
\cline { 2 - 5 } & SPSO & $7,9,14,32,3$ \\
& {$[33]$} & 136.3 & 0.942 \\
\cline { 2 - 5 } & MPSO & $\begin{array}{c}7,9,14,32, \\
37\end{array}$ & 136.3 & 0.940 \\
\hline
\end{tabular}

A Comparison between the MPSO results and previous researches using different form of swarm algorithms, is presented in table 3 . The proposed modifications added to the typical swarm technique achieved $136.36 \mathrm{KW}$. The losses calculation of the suggested tie configurations in [25-26] are recalculated using python/IPSA, and the results are illustrated in table 3. The suggested MPSO, which is based on a filtered random position selection surpass the typical swarm used in both [25], [26] not only in losses reduction, but also in the excitation time. MPSO saved $56.7 \mathrm{KW}$ of losses during 17.5 seconds compared to $43.2 \mathrm{KW}$ of losses reduction by the typical swarm in [25] during 25 seconds. Also, the proposed algorithm MPSO, achieved more losses reduction than Binary Particle Swarm used in [35], and suggest the same tie switches given by Selective Particle Swarm (SPSO) in [33], achieving the same losses reduction. This in turn confirms that the added modifications improved both the power losses reduction and the computational time.

\section{CONCLUSION}

In this paper, the MPSO is proposed for network reconfiguration for losses reduction and in turns voltage improvement. The modification added to the typical PSO technique has accelerated the computational time to get an optimum solution.33 IEEE network was used for validation using IPSA software. MPSO reduced the power losses by $29.68 \%$ during 17.5 seconds. The results are compared to other versions of swarm at static load, and it has been shown that the algorithm gives better losses reduction than typical PSO and in less computational time, and give the same percentage of losses reduction given by SPSO. In this paper, the algorithm is tested for 50 trials to select the swarm size and the maximum iterations number required for optimum solution. The proposed technique will be tested on variable load for real network implementation.

\section{REFERENCES}

[1] K.M Valsamma, "Smart Grid as a Desideratum in the Energy Landscape: Key Aspects and Challenges", in Proc. International Engineering Education: Innovative Practices and Future Trends (AICERA), IEEE ,2012, Kottayam, pp.1-6.

[2] X. Fang, S.Misra and G.Xue,"Smart Grid - The New and Improved Power Grid: A Survey", in Proc. communications surveys and Tutorials,2012, Vol.14, No.4, pp.944-980.

[3] L.Tang, F.Yang and J.Ma, "A survey on distribution system feeder reconfiguration: objectives and solutions", in Proceedings of Innovative Smart Grid technology Asia (ISGT), Kuala Lumpur, May, 2014, IEEE, pp.62-67.

[4] B .Akduman , B.Turkay and A.S.Uyar., "Service restoration in distribution systems using an evolutionary algorithm", Proceedings of 7 th Mediterranean conference and exhibition on power generation, transmission, distribution and energy conversion,November,2010, Agia Napa, IET, pp 1-9.

[5] H.Esmailian and R. Fadaeinedjad, "Energy loss minimization in distribution systems utilizing an enhanced reconfiguration method integrating distributed generation", Systems Journal, IEEE, August, 2014, pp.1-10.

[6] O.Duque and D. Morinigo, "Load restoration in electric distribution networks using a metaheuristic technique", Proceedings of Electrotechnical Conference, MELECON, Mediterranean, Malaga, May, 2006, IEEE, pp.1040-1043.

[7] L.Tang, F.Yang and J.Ma, "A survey on distribution system feeder reconfiguration: objectives and solutions", in Proceedings of Innovative Smart Grid technology Asia (ISGT), Kuala Lumpur, May, 2014, IEEE, pp.62-67.

[8] Z.A. Vale,B.Canizes, J.Soares,P.Oliveira,T. Sousa, and T. Pinto, "Logic programming and fuzzy monte carlo for distribution network reconfiguration", in Proceedings of 16th International Conference on Intelligent System Application to Power Systems, Hersonissos , September,2011 ,IEEE, pp.1-6.

[9] J. Li and J. Zhao, "Combining differential evolution algorithm with biogeography based optimization algorithm for reconfiguration of distribution network", in Proceedings of International Conference on Power System Technology, November, 2012, Auckland, IEEE, pp.1-6.

[10] G.Chicco and A.Mazza, "An overview of the probability based methods for optimal electrical distribution system reconfiguration", in Proceedings of 4th International Symposium on Electrical and Electronics Engineering (ISEEE), October, 2013, Galati, pp.1-10.

[11] S. Kirkpatrick, C. D. Gelatt, and M. P. Vecchi , "Optimization by simulated annealing”, Science, vol.220, no. (4598), pp.671-680, May, 1983.

[12] Song Nie, X.Fu, P. Li, F. Gao, Ch.Ding, Hao Yu, and Ch.Wang, "Analysis of the impact of DGs on distribution network reconfiguration using open DSS", in Proceedings of Innovative Smart Grid Technologies (ISGT) Asia, May, 2012, Tianjin, IEEE, pp.1-5.

[13] W.Gang ,L.Li, and T. Zhang, "An improved ant colony search algorithm for reconfiguration of distribution network with distributed generations innovative smart grid technologies", in Proceedings of Innovative Smart Grid Technologies (ISGT) Asia, May, 2012, Tianjin, IEEE, pp.1-4.

[14] V. Farahani, B.Vahidi, and H. Abyaneh , "Reconfiguration and capacitor placement simultaneously for energy Loss Reduction Based on an improvedReconfiguration Method", IEEE Transactions on power systems, vol.27, no.(2), pp. 587 - 595, May, 2012.

[15] A. Swarnkar, N.Gupta and K.R.Niazi, "Distribution network reconfiguration using population based AI techniques: A Comparative 
Analysis", in Proceedings of Power and Energy Society General Meeting, July, 2012, San Diego, IEEE, pp.1-6.

[16] P.Kayale, S.Chanda and C.K.Chanda, "An ANN based network reconfiguration approach for voltage stability improvement of distribution network", in Proceedings of International Conference on power and Energy System, December, 2011, IEEE, Chennai ,pp.1-7.

[17] Z.W.Geem, J.Hoon Kim, G. Loganathan, “Anew heuristic optimization algorithm: harmony search”, simulation, vol.76, pp.60-68, August 2001.

[18] R.S.Rao , K. Ravindra, K. Satish, and S. V. L. Narasimham, "Power loss minimization in distribution system using network reconfiguration in the presence of distributed generation", Transactions on Power system, vol.28, no. (1), pp.1-9, February, 2013.

[19] D.Rani, N. Subrahmanyam, and M. Sydulu,"Improved music based harmony search algorithm for optimal network reconfiguration", in Proceedings of India Conference, December, 2012, Kochi, IEEE, pp.1030-1035.

[20] H.Savari and K. Zamanifar, "A self adaptive harmony search algorithm for engineering and reliability problems", in proceedings of the 2 nd International conference on computational intelligence, Modeling and Simulation,September,2010, Bali, pp.59-64.

[21] D.Rani, N. Subrahmanyam and M. Sydulu, "Self adaptive harmony search algorithm for optimal network reconfiguration", in Proceedings of Power and Energy Conference (PECI) at Illinois, February/March,2014, Champaign, IEEE, pp.1-6.

[22] M.Dorigo, "Optimization learning and natural algorithms", $\mathrm{PhD}$, dissertation, Dep. Elec. Eng. University of Milano, Italy, 1992.

[23] J.Kennedy and R.Eberhart, "Particle swarm optimization", in Proceeding of the fourth International Conference on Neural Networks, November/December, 1995, Perth, pp.1942-1948.

[24] X.S.Yang and S. Deb "Engineering optimization by cuckoo search", Int. J. Mathematical Modeling and Numerical Optimization, vol. 1, no. (4), pp.330-343, December, 2010. Available: http://arxiv.org/pdf/1005.2908.pdf. [Acssessed:October.28.2015].

[25] M.Nasir, N. M. Shahrin, Z. H. Bohari, M. F. Sulaima, and M. Y. Hassan,"A Distribution network reconfiguration based on PSO considering DGs sizing and allocation evaluation for voltage profile improvement", in Proceedings of Research and Development, December, 2014, Batu Ferringhi, IEEE, pp.1-6.

[26] W.Dahalan and H. Mokhlis, "Network reconfiguration for loss reduction with distributed generations using PSO", in Proceedings of International conference on Power and Energy, December,2012, Kota Kinabalu, IEEE, pp.823-828.

[27] I.Sanz et al., "Multi-layer agent based architecture for smart grid reconfiguration", in Proceedings of 40th Annual Conference of Industrial Electronic Society (ICON), October/November, 2014, Dallas IEEE, pp. 3570 - 3576.

[28] J.Kennedy and R.Eberhart, " A discrete binary version of the particle swarm optimization algorithm", in proceedings of the World Multi conference on Systemic, Cybernetics and informatics, October, 1997, Orlando,pp.4104-4109.

[29] C.CoelloCoello, G. Toscano Pulid and M. S. Lechuga , "Handling multi objectives with particle swarm optimization", congress of evolutionary computation, vol.8, no.(3),June, 2004.

[30] A.Arya, Y. Kumar , and M. Dubey,"Reconfiguration of electric distribution network using modified particle swarm optimization", International Journal of Computer applications, Vol.34, No.6, November, 2011, pp.54-62.

[31] S.R.Tuldhar, J. Singh, and W.Ongsakul, , "A multi objective network reconfiguration of distribution network with solar and wind distributed generation using NSPSO", in Proceedings of International Conference and Utility Exhibition on Green Energy for Sustainable Development (ICUE), March,2014, Pattaya,IEEE,pp.1-6.

[32] T.Khalil and A.Gorpinich, "Reconfiguration of loss reduction in distribution system using selective particle swarm optimization", International journal of Multidisciplinary Sciences and Engineering, vol.3, no. (6), pp.1-4, 2012.

[33] A.Tandon and D.Saxena, "A comparative Analysis of SPSO and BPSO for power loss minimization in distribution system using network reconfiguration", in Proceedings of International Conference on
Innovative Applications of Computational Intelligence on Power Energy and Controls with their impact on Humanity November,2014.IEEE,pp.226-232.

[34] S.K. Goswami and S.K. Basu," A new algorithm for the reconfiguration of distribution feeders for loss minimization", IEEE Transactions on Power Delivery, Vol. 7, No. 3, Jul 1992

[35] A.Y.Abdelaziz, $\quad$ F.M.Mohamed, S.F.Mekhamer,M.A.L.Badr," Distribution Systems Reconfiguration using a modified particle swarm optimization",Electrical power system and Research , El sevier, vol. 79 (11), pp. 1521-1530, Nov. 2009Table 3 line data for 33IEEE network.

[36] http://www.ipsa-power.com/

[37] J.Giu, O Penangsang, and R.Wibowo,"Implementation and validation of different reconfiguration strategies between HAS and PSO for loss reduction" International journal of Innovative Research in Electrical, Electronic Instrumentation and control Engineering, vol.1, pp.32-37, May 2013.

APPENDIX [33-33 IEEE line data

\begin{tabular}{|c|c|c|c|c|c|c|}
\hline & From & To & $\begin{array}{c}\mathrm{R} \\
(\Omega)\end{array}$ & $\begin{array}{c}X \\
(\Omega)\end{array}$ & $\begin{array}{c}\mathrm{P} \\
(\mathrm{kW})\end{array}$ & $\begin{array}{c}\mathrm{Q} \\
(\mathrm{kVAR})\end{array}$ \\
\hline $\mathrm{S} 1$ & 1 & 2 & 0.0922 & 0.047 & 100 & 60 \\
\hline $\mathrm{S} 2$ & 2 & 3 & 0.493 & 0.2512 & 90 & 40 \\
\hline S3 & 3 & 4 & 0.3661 & 0.1864 & 120 & 80 \\
\hline S4 & 4 & 5 & 0.3811 & 0.1941 & 60 & 30 \\
\hline S5 & 5 & 6 & 0.8190 & 0.7070 & 60 & 20 \\
\hline S6 & 6 & 7 & 0.1872 & 0.6188 & 200 & 100 \\
\hline S7 & 7 & 8 & 0.7115 & 0.2351 & 200 & 100 \\
\hline S8 & 8 & 9 & 1.0299 & 0.7400 & 60 & 20 \\
\hline S9 & 9 & 10 & 1.044 & 0.7400 & 60 & 20 \\
\hline $\mathrm{S} 10$ & 10 & 11 & 0.1967 & 0.0651 & 45 & 30 \\
\hline S11 & 11 & 12 & 0.3744 & 0.1298 & 60 & 35 \\
\hline $\mathrm{S} 12$ & 12 & 13 & 1.4680 & 1.1549 & 60 & 35 \\
\hline S13 & 13 & 14 & 0.5416 & 0.7129 & 120 & 80 \\
\hline S14 & 14 & 15 & 0.5909 & 0.5260 & 60 & 10 \\
\hline S15 & 15 & 16 & 0.7462 & 0.5449 & 60 & 20 \\
\hline S16 & 16 & 17 & 1.2889 & 1.7210 & 60 & 20 \\
\hline S17 & 17 & 18 & 0.7320 & 0.5739 & 90 & 40 \\
\hline S18 & 2 & 19 & 0.1640 & 0.1565 & 90 & 40 \\
\hline S19 & 19 & 20 & 1.5042 & 1.3555 & 90 & 40 \\
\hline S20 & 20 & 21 & 0.4095 & 0.4784 & 90 & 40 \\
\hline $\mathrm{S} 21$ & 21 & 22 & 0.7089 & 0.9373 & 90 & 40 \\
\hline $\mathrm{S} 22$ & 3 & 23 & 0.4512 & 0.3084 & 90 & 50 \\
\hline S23 & 23 & 24 & 0.8980 & 0.7091 & 420 & 200 \\
\hline S24 & 24 & 25 & 0.8980 & 0.7071 & 420 & 200 \\
\hline S25 & 6 & 26 & 0.2031 & 0.1034 & 60 & 25 \\
\hline S26 & 26 & 27 & 0.2842 & 0.1474 & 60 & 25 \\
\hline S27 & 27 & 28 & 1.0589 & 0.9338 & 60 & 20 \\
\hline S28 & 28 & 29 & 0.8043 & 0.7006 & 120 & 70 \\
\hline S29 & 29 & 30 & 0.5074 & 0.2585 & 200 & 600 \\
\hline S30 & 30 & 31 & 0.9745 & 0.9629 & 150 & 70 \\
\hline S31 & 31 & 32 & 0.3105 & 0.3619 & 210 & 100 \\
\hline S32 & 32 & 33 & 0.341 & 0.5302 & 60 & 40 \\
\hline S33 & 8 & 21 & 2.00 & 2.00 & - & - \\
\hline S34 & 9 & 15 & 2.00 & 2.00 & - & - \\
\hline S35 & 12 & 22 & 2.00 & 2.00 & - & - \\
\hline S36 & 18 & 33 & 0.500 & 0.500 & - & - \\
\hline S37 & 25 & 29 & 0.500 & 0.500 & - & - \\
\hline
\end{tabular}


